\title{
Expanding intestinal stem cells in culture
}

\author{
Cell Research (2015) 25:995-996. doi:10.1038/cr.2015.83; published online 14 July 2015
}

\begin{abstract}
Culturing intestinal stem cells into 3D organoids results in heterogeneous cell populations, reflecting the in vivo cell type diversity. In a recent paper published in Nature, Wang et al. established a culture condition for a highly homogeneous population of intestinal stem cells.
\end{abstract}

Adult stem cells are the main drivers of tissue maintenance and regeneration. Development of in vitro culture methods of adult stem cells is paramount to a better understanding of tissue homeostasis as well as stem cell biology. Stem cell culture technologies also hold great promise for clinical applications such as disease modeling and regenerative medicine. Over the past decade, significant progress has been made for establishing culture methods for tissue stem cells directly from healthy or diseased mouse and human organs. Now Wang et al. [1] developed a new method for the specific expansion of intestinal stem cells.

Intestinal epithelium is continuously replenished by resident adult stem cells residing at the base of the crypts. In vitro expansion of intestinal epithelia was previously achieved by recreating in vivo crypt niche conditions of intestinal stem cells [2-4]. When a single intestinal stem cell is cultured in a three-dimensional (3D) matrix, the stem cell grows into an ever-expanding organoid that mirrors the architecture of in vivo intestine (Figure 1). The organoid culture (also known as a mini-gut) can be expanded for more than one and half years while maintaining a stable genome [3]. These organoids faithfully recapitulate the physiology and functionality of the in vivo epithelium.
Indeed, organoids derived from a single intestinal stem cell were successfully transplanted into the damaged colons of multiple mice [5].

Organoids resemble in vivo intestinal epithelia that contain low numbers of adult stem cells at the base of extruding crypts. Thus, only a small fraction of stem cells exist within organoids (Figure 1), which may set a potential barrier in understanding molecular and cellular properties of stem cells themselves. The addition of two small molecules reinforcing Wnt and Notch signals to the culture medium resulted in a substantial increase of stem cell percentages in human and mouse small intestinal organoids [6]. However, it remained unclear whether the genome stability of the stem cell under this condition was retained during long-term expansion and whether the method could also be applied to human colonic organoids.

In a paper recently published in Nature, Wang and colleagues describe a condition to support long-term and highly homogeneous preparations of stem cells from human fetal and adult small intestine and colon [1]. Single cell suspensions were seeded on a feeder layer of mouse fibroblasts. The stem cells grew into 2-dimensional (2D) colonies that consisted entirely of highly clonogenic stem cells (Figure 1). The colonies of intestinal stem cells retained self-renewal capability and maintained genome stability over many passages. Only under a specific condition using air-liquid interface, the stem cells started to generate all types of differentiated cells present in the intestine, thus partially mimicking the anatomy of the in vivo intestine.
Organoid culture is conducted in a laminin-rich 3D matrix (Matrigel), within which a stem cell rapidly selfrenews and eventually creates a closed, cystic 3D structure [7]. Stem cells grown under Wang's condition, by contrast, continue to propagate as flat $2 \mathrm{D}$ colonies on the feeder layer and do not differentiate [1]. Since the growth factor additions are nearly identical between the two conditions (containing Wnt agonist R-spondin, EGF, BMP antagonist and TGF- $\beta$ inhibitor), the matrix as well as the focal production of Wnt3 by Paneth cells in organoids $[7,8]$ might be a critical factor for the difference between the two systems.

Wang et al. [1] applied this culture model to probe molecular properties of intestinal stem cells. It was previously reported that adult intestinal stem cells carry information of commitment to region-specific differentiation along intestinal tract by analyzing gene expression of organoid system [9]. Utilizing clonal cultures, Wang et al. proved that this region-specific commitment in intestinal stem cells was intrinsically programmed from the fetus (20-21 weeks). In addition, the authors compared the transcriptome profile of intestinal stem cell cultures with those of stem cells derived from epidermis, airway, and others. Taken together, these studies demonstrate that the culture system by Wang et al. offers potential for an in-depth study on molecular and functional features of adult stem cells.

Wang et al. [1] showed that clonal stem cells yield all types of differentiated cells upon induction differentiation. However, it remains unknown whether the dynamic turnover of epithelia oc- 


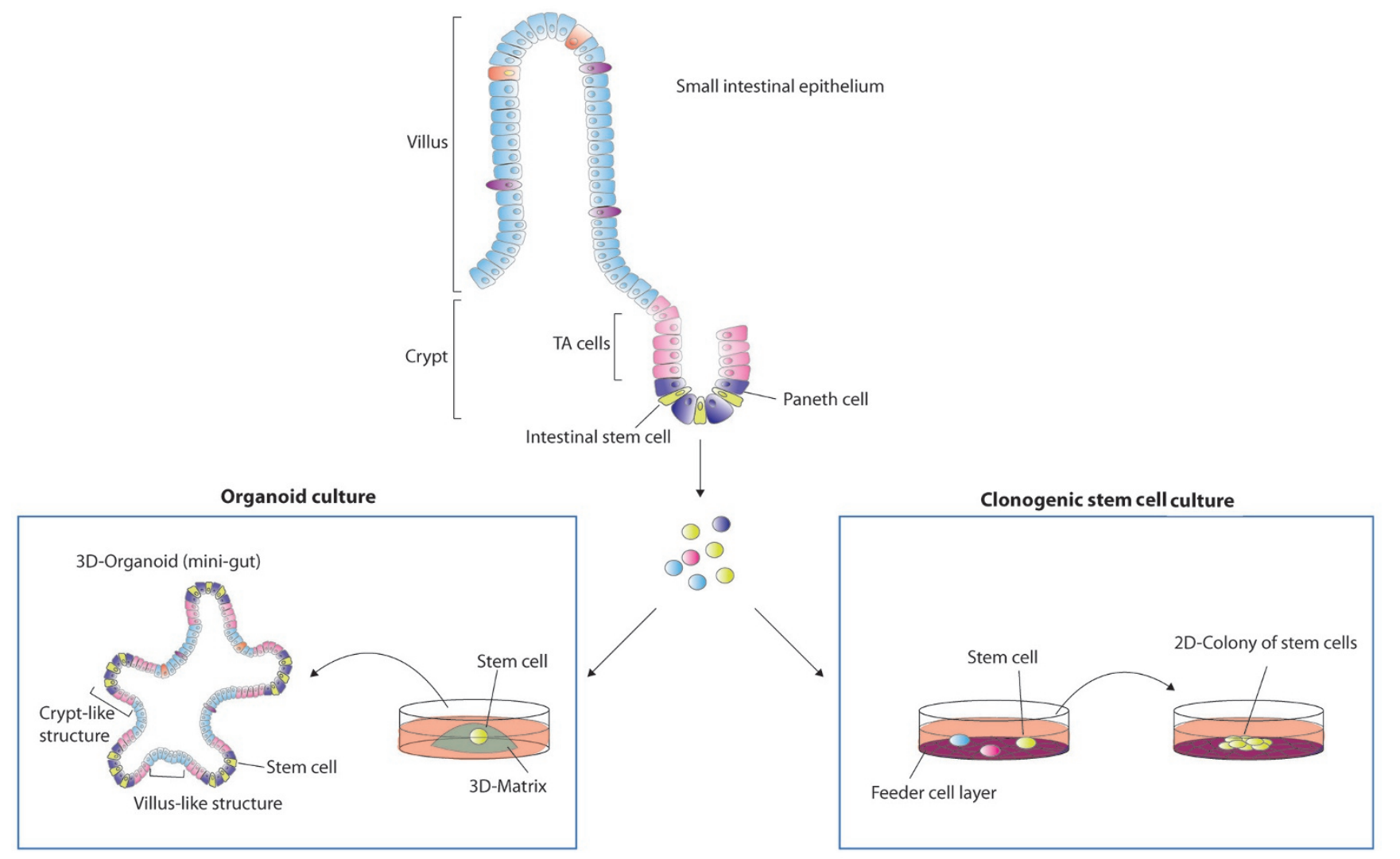

Figure 1 Schematic representation of intestinal stem cell cultures. Intestinal epithelium consists of villus and crypt compartments. Villus comprises differentiated cells, while crypt consists of stem cells, transit-amplifying (TA) cells, and Wnt-secreting paneth cells. (Left) A single intestinal stem cell in a 3D-matrix grows into an ever-expanding organoid which comprises all types of cells present in intestine. Organoids recapitulate the physiology and architecture of in vivo epithelium. (Right) Suspended single cells from intestinal epithelium grow into colonies consisting of clonogenic stem cells on a feeder layer of fibroblast. Stem cell colonies maintain the capability of selfrenewal over many passages.

curs in these differentiated conditions and, even so, how closely this turnover rate might resemble the in vivo situation. Organoids, on the contrary, faithfully represent the in vivo architecture, physiology and the full complement of cell populations [7]. Thus, the two methods will complement each other for research on adult stem cells and tissue homeostasis. For a future clinical application, it will be interesting to find out whether clonogenic stem cells can be functionally integrated into damaged mouse intestine.

\section{Inha $\mathrm{Heo}^{1}$, Hans Clevers ${ }^{1}$}

${ }^{1}$ Hubrecht Institute, Royal Academy of Arts and Science (KNAW) and University Medical Centre Utrecht, Uppsalalaan 8, 3584CT, Utrecht, The Netherlands

Correspondence: Hans Clevers

E-mail: h.clevers@hubrecht.eu

\section{References}

1 Wang X, Yamamoto Y, Wilson LH, et al. Nature 2015; 522:173-178.

2 Sato T, Vries RG, Snippert HJ, et al. Nature 2009; 459:262-265.

3 Sato T, Stange DE, Ferrante M, et al. Gas- troenterology 2011; 141:1762-1772.

4 Jung P, Sato T, Merlos-Suarez A, et al. Nat Med 2011; 17:1225-1227.

5 Yui S, Nakamura T, Sato T, et al. Nat Med 2012; 18:618-623.

6 Yin X, Farin HF, van Es JH, et al. Nat Methods 2014; 11:106-112.

7 Sato T, Clevers H. Science 2013; 340:11901194.

8 Farin HF, Van Es JH, Clevers H. Gastroenterology 2012; 143:1518-1529.e7.

9 Middendorp S, Schneeberger K, Wiegerinck CL, et al. Stem Cells 2014; 32:1083-1091. 